\title{
The Sobolev stability threshold for 2D shear flows near Couette
}

\author{
Jacob Bedrossian, Vlad Vicol, and Fei Wang
}

\begin{abstract}
We consider the 2D Navier-Stokes equation on $\mathbb{T} \times \mathbb{R}$, with initial datum that is $\varepsilon$-close in $H^{N}$ to a shear flow $(U(y), 0)$, where $\|U(y)-y\|_{H^{N+4}} \ll 1$ and $N>1$. We prove that if $\varepsilon \ll \nu^{1 / 2}$, where $\nu$ denotes the inverse Reynolds number, then the solution of the Navier-Stokes equation remains $\varepsilon$-close in $H^{1}$ to $\left(e^{t \nu \partial_{y y}} U(y), 0\right)$ for all $t>0$. Moreover, the solution converges to a decaying shear flow for times $t \gg \nu^{-1 / 3}$ by a mixing-enhanced dissipation effect, and experiences a transient growth of gradients. In particular, this shows that the stability threshold in finite regularity scales no worse than $\nu^{1 / 2}$ for 2D shear flows close to the Couette flow. July 17, 2018.
\end{abstract}

\section{Introduction}

A fundamental problem in the field of hydrodynamic stability is to assess the stability of shear flows in the (2D or 3D) Navier-Stokes equations at high Reynolds number $(\nu \rightarrow 0)$; see e.g. the texts [DR81, SH01, Yag12] and the references therein.

In this paper we consider the incompressible 2D Navier-Stokes equations

$$
\begin{aligned}
\partial_{t} \widetilde{v}+\widetilde{v} \cdot \nabla \widetilde{v}-\nu \Delta \widetilde{v}+\nabla \widetilde{p} & =0, \quad \nabla \cdot \widetilde{v}=0, \\
\widetilde{v}(t=0) & =\widetilde{v}_{\text {in }}
\end{aligned}
$$

on the domain $\Omega=\{(x, y) \in \mathbb{T} \times \mathbb{R}\}$, where $\nu$ denotes the inverse Reynolds number. Here $\mathbb{T}$ is the periodized interval $[0,1]$. The initial datum $\widetilde{v}_{\text {in }}$ is taken to be a small perturbation of a shear flow profile $(U(y), 0)$, and we write

$$
\widetilde{v}_{\text {in }}(x, y)=(U(y), 0)+\bar{v}_{\text {in }}(x, y) .
$$

If $\bar{v}_{\text {in }} \equiv 0$, the solution of the 2D Navier-Stokes equation (1.1a)-(1.1b) is given by the heat evolution of the shear profile, i.e.

$$
(\bar{U}(t, y), 0)=\left(e^{\nu t \partial_{y y}} U(y), 0\right) .
$$

In this paper we are concerned only with shears $U(y)$ which satisfy $\left\|\partial_{y} U-1\right\|_{H^{s}} \ll 1$ for $s$ large enough (see Theorem 1.1 below for precise requirement) and hence are close to the Couette flow $U(y)=y$. For small (but nontrivial) $\bar{v}_{\text {in }}$, we look for a solution $\widetilde{v}$ as a perturbation of this decaying shear profile and define

$$
\widetilde{v}(t, x, y)=(\bar{U}(t, y), 0)+\bar{v}(t, x, y)
$$

with the equations satisfied by $\bar{v}$ being obtained from (1.1a)-(1.1b) as

$$
\begin{aligned}
\bar{v}_{t}+\bar{U} \partial_{x} \bar{v}+\bar{v} \cdot \nabla \bar{v}-\nu \Delta \bar{v}+\left(\bar{U}^{\prime} \bar{v}^{y}, 0\right)+\nabla p & =0, \quad \nabla \cdot \bar{v}=0, \\
\bar{v}(t=0) & =\bar{v}_{\text {in }} .
\end{aligned}
$$


In (1.3a) we have used the notation $\bar{U}^{\prime}=\partial_{y} \bar{U}$, and $\bar{v}^{y}$ for the second, i.e. $y$, component of the vector field $\bar{v}$. Taking the curl of equation (1.3a) and denoting

$$
\omega=\operatorname{curl} \bar{v},
$$

we obtain the vorticity formulation of the perturbed Navier-Stokes equation

$$
\begin{aligned}
\omega_{t}+\bar{U} \partial_{x} \omega+\bar{v} \cdot \nabla \omega & =\bar{U}^{\prime \prime} \partial_{x} \psi+\nu \Delta \omega \\
\Delta \psi & =\omega \\
\bar{v} & =\nabla^{\perp} \psi \\
\omega(t=0) & =\omega_{\text {in }},
\end{aligned}
$$

where we have used the notation $\bar{U}^{\prime \prime}=\partial_{y y} \bar{U}$ and $\nabla^{\perp}=\left(-\partial_{y}, \partial_{x}\right)$. In (1.4b) the stream function $\psi$ is taken to have zero mean over $\Omega$, so that we may write $\psi=-(-\Delta)^{-1} \omega$ and $\bar{v}=-\nabla^{\perp}(-\Delta)^{-1} \omega$.

1.1. Statement and discussion of the main result. The purpose of this paper is to study the long-time dynamics of the perturbation $\omega$ solving (1.4) in the high Reynolds number limit $\nu \rightarrow 0$.

Our main goal is to estimate the stability threshold for the solution $\bar{U}(t, y)$, and in particular, to determine how it scales with respect to $\nu$. That is, given an $N>1$, try to find the smallest $\gamma>0$ such that $\left\|\omega_{\text {in }}\right\|_{H^{N}}=\varepsilon \ll \nu^{\gamma}$ implies that the perturbation $\omega$ remains small in $L^{2} \cap H^{-1}$, and to determine the dynamics of such stable solutions. We shall see that stability uniformly in $\nu$ in $H^{s}$ for any $s>0$ is necessarily false as the solution undergoes a large transient growth in these norms due to the mixing caused by the shear.

At high Reynolds number, in 3D experiments and computer simulations, nonlinear instability is often observed at a lower Reynolds numbers than what is predicted by linear theory. This is usually referred to as subcritical transition [SH01, Yag12]. In some cases, such as (1.4), the flow is linearly stable at all Reynolds numbers, but the nonlinear stability threshold might be decreasing as $\nu \rightarrow 0$, resulting in instability at a finite Reynolds number in any experiment or simulation. Hence, given a norm $\|\cdot\|_{X}$, the goal is to determine a $\gamma=\gamma(X)$ such that (naturally we do not know a priori that it is a power law),

$$
\begin{aligned}
& \left\|\omega_{\text {in }}\right\|_{X} \lesssim \nu^{\gamma} \Rightarrow \text { stability } \\
& \left\|\omega_{\text {in }}\right\|_{X} \gg \nu^{\gamma} \Rightarrow \text { possible instability. }
\end{aligned}
$$

In the applied math and physics literature, $\gamma$ is sometimes referred to as the transition threshold.

The minimal value of $\gamma$ is expected to depend non-trivially on the norm $X$. For example, in the numerical experiments of [RSBH98] on 3D Couette flow, it is estimated that "rough" initial perturbations (e.g. weaker $X$ ) result in a higher $\gamma$. In the case of $3 D$ Couette flow, it was shown in [BGM15a, BGM15b] that $\gamma=1$ for $X$ taken as Gevrey- $m$ with $m<2$, and that $\gamma \leq 3 / 2$ for $X=H^{s}$ for $s>7 / 2$ in [BGM15c] (the latter estimate is consistent with the numerical estimation of $31 / 20$ given in [RSBH98]).

However, for the $2 D$ Couette flow, it was shown in [BMV16] that in fact $\gamma=0$ for $X$ taken as Gevrey- $m$ with $m<2$. That is, for initial perturbations taken sufficiently smooth, the Couette flow is uniformly stable at high Reynolds number and there is no subcritical transition. It was shown earlier in [BM13] that the Couette flow is also nonlinearly stable (in a suitable sense) for the 2D Euler equations, the case $\nu=0$, for such sufficiently smooth Gevrey perturbations.

In this paper, we estimate the stability threshold in 2D, now in Sobolev regularity (as opposed to in a Gevrey class [BMV16]). As in 3D, we could expect the stability threshold in Sobolev regularity to be worse than in a Gevrey class (or at least, our estimate of the stability threshold). Indeed, it 
follows from [LZ11] for that topologies $X$ which are weaker than $H^{3 / 2}$, we should expect $\gamma>0$ even for the Couette flow. The stability threshold we estimate is $\gamma \leq 1 / 2$. Our main result is:

THEOREM 1.1. Let $N>1,0<\nu \leq 1$, and $C \geq 1$ be a sufficiently large constant depending only on $N$ (in particular, it is independent of $\nu$ ). Consider a shear flow $U=U(y)$ such that

$$
\left\|U^{\prime}-1\right\|_{H^{s}(\mathbb{R})}+\left\|U^{\prime \prime}\right\|_{H^{s}(\mathbb{R})}=\delta \leq C^{-1}
$$

for some $s \geq 2+N$ and $\delta$ independent of $\nu$. Assume that the initial perturbation obeys

$$
\left\|\omega_{\text {in }}\right\|_{H^{N}(\Omega)}+\left\|\bar{v}_{\text {in }}\right\|_{L^{2}(\Omega)}=\varepsilon \leq C^{-1} \nu^{1 / 2} .
$$

Then the global in time solution $\omega$ to (1.4a)-(1.4d) obeys

$$
\begin{aligned}
\|\bar{v}\|_{L^{\infty}\left(0, \infty ; L^{2}(\Omega)\right)}+\left\|\omega_{0}\right\|_{L^{\infty}\left(0, \infty ; H^{N}(\Omega)\right)}+\nu^{1 / 2}\left\|\nabla \omega_{0}\right\|_{L^{2}\left(0, \infty ; H^{N}(\Omega)\right)} & \leq C \varepsilon \\
\|\omega \circ(x+t \bar{U}(t, y), y)\|_{L^{\infty}\left(0, \infty ; H^{N}(\Omega)\right)} & \leq C \varepsilon
\end{aligned}
$$

and we have the enhanced dissipation estimate

$$
\left\|\omega_{\neq}\right\|_{L^{2}\left(0, \infty ; H^{N}(\Omega)\right)} \leq C \varepsilon \nu^{-1 / 6} .
$$

In (1.6)-(1.7) we have denoted by $\omega_{\neq}$the projection of $\omega$ onto its nonzero Fourier modes with respect to $x$, and by $\omega_{0}$ the projection of $\omega$ onto the zero Fourier mode with respect to $x$ (see also (1.10)).

REMARK 1.2. Estimate (1.7) encodes that the time-scale on which the deviation from the mean in $x$ is decaying is much faster than the heat equation time-scale (which would be $\nu^{-1 / 2}$ ). This "enhanced dissipation" effect is the key to this work, and is discussed further below in Section 1.2.

REMARK 1.3. Notice that Theorem 1.1 is neither stronger nor weaker than the results of [BMV16]. In [BMV16], much stronger regularity hypotheses are taken, however, $\varepsilon$ can be chosen independently of $\nu$, whereas in Theorem 1.1, the data is permitted to be much rougher but is instead required to be much smaller, specifically $\varepsilon \lesssim \nu^{1 / 2}$.

REMARK 1.4. The composition in (1.6b) looks slightly non-standard, but note that $\bar{U}(t, y)$ is essentially constant (in time) until $t \gtrsim \nu^{-1}$, at which point $\omega_{\neq}$is essentially zero by (1.7).

REMARK 1.5. The regularity assumption $N>1$ arises due to the fact that this is the smallest Sobolev exponent which guarantees the local in time well-posedness of the underlying 2D Euler equation $(\nu=0)$. Since our constants are all independent of $\nu$ (which may be taken arbitrarily small), such a requirement on the size of $N$ is arguably natural.

REMARK 1.6. Using techniques from [BMV16], one can slightly weaken the regularity requirement (1.5) via local parabolic smoothing. Specifically, if one can decompose $\omega_{\text {in }}=\omega_{\text {in }}^{R}+\omega_{\text {in }}^{S}$ such that for an $\eta>0$ we have $\left\|\omega_{\text {in }}^{S}\right\|_{H^{N}(\Omega)}+\nu^{1 / 2+\eta}\left\|\omega_{\text {in }}^{R}\right\|_{L^{2}}+\left\|\bar{v}_{\text {in }}\right\|_{L^{2}(\Omega)}=\varepsilon \ll_{\eta, N} \nu^{1 / 2}$, then the conclusions of Theorem 1.1 apply, except that the inequalities (1.6) and (1.7) hold over $(1, \infty)$.

REMARK 1.7. Naturally, the value $\gamma=1 / 2$ is much lower than for 3D Couette in Sobolev spaces (in 3D, the estimated value is $\gamma \leq 3 / 2$ [BGM15c], consistent also with numerics [RSBH98]). The major differences between $2 \mathrm{D}$ and $3 \mathrm{D}$ are due to: the linearized $3 \mathrm{D}$ Couette flow induces vortex stretching and a 3D non-normal transient growth known as the lift-up effect; the class of $x$-independent flows is much larger in 3D; and the nonlinearity has a much more problematic "nonlinear resonance" structure in 3D (see [BGM15a] for more discussion). It seems difficult to lower $\gamma$ here without confronting the main nonlinear source of regularity losses encountered in the case of Gevrey initial datum [BM13, BMV16]. See Subsection 1.2 below for more discussion. 
In order to fix the main ideas of the proof, we first prove Theorem 1.1 in the case of the Couette flow $U(y)=y$ (see Section 2 below). The advantage here is that $\bar{U}(t, y)=y$ for all $t>0$, and thus the change of coordinates we use to account for the fast action of the shear flow is trivial (cf. (2.1)). Moreover, the case $U(y)=y$ has the advantage that the linearized Navier-Stokes equations lack the $\bar{U}^{\prime \prime}$ velocity term (see e.g. the right side of (1.4a)) and that the Biot-Savart law is a Fourier multiplier in the new variables. These make the proof of Theorem 1.1 in the case of Couette more transparent. At the linear level, the main effects we take advantage of are inviscid damping and enhanced dissipation (see Section 1.2 below).

In proving Theorem 1.1 for the more general class of shear flows which are "close to Couette", we use the same main ideas as in the Couette case (e.g. the same norm), but we are faced with a number of technical difficulties due to a more complicated change of coordinates (cf. (3.3)) which needs to be adapted to the decaying shear. For example, we need to better quantify the transfer of kinetic energy from the non-zero modes (with respect to $x$ ) to the zero one. At the technical level, we also need to consider a number of new commutators between the Fourier multipliers that define the norm and multiplication operators with functions of $y$. These details are given in Section 3.2.

1.2. The linearized problem: inviscid damping and enhanced dissipation. Before addressing the nonlinear problem, it is important to understand the properties of the linearized problem in the case $U(t, y)=y$, which we review here; see also [BMV16] for similar discussions. In the case $U(t, y)=y$, the linearization of (1.4) becomes

$$
\begin{aligned}
\partial_{t} \omega+y \partial_{x} \omega & =\nu \Delta \omega \\
\Delta \psi & =\omega .
\end{aligned}
$$

These equations were first solved by Kelvin in [Kel87]. The solution is given, in Fourier space, by

$$
\widehat{\omega}(t, k, \eta)=\widehat{\omega}_{\text {in }}(k, \eta+k t) \exp \left[-\nu \int_{0}^{t} k^{2}+|\eta-k(\tau-t)|^{2} d \tau\right] .
$$

From this formula, one verifies the enhanced dissipation effect: for some universal $c>0$ there holds

$$
\left\|\omega_{\neq}(t)\right\|_{L^{2}} \lesssim\left\|\omega_{\text {in }}\right\|_{L^{2}} e^{-c \nu t^{3}}
$$

which explains the accelerated time-scale (1.7). Physically, the fast mixing of the Couette flow is sending information to high frequencies linearly in time, enhancing the viscous damping at the corresponding rate. This mixing-enhanced dissipation effect or related mechanisms have been studied in many works on linear equations, for example, in [CKRZ08, GGN09, Zla10, BW13, VDPS15, BCZGH15, BCZ15] and in the physics literature [Lun82, RY83, DN94, LB01, BL94]. This effect implies that solutions strongly converge to a slowly decaying shear flow after times like $t \gtrsim \nu^{-1 / 3}$.

A related, but more subtle effect, is that of inviscid damping, first noticed by Orr in [Orr07]. For this, note that

$$
\begin{aligned}
\widehat{\psi}(t, k, \eta) & =\frac{\widehat{\omega}_{\text {in }}(k, \eta+k t)}{k^{2}+\eta^{2}} \exp \left[-\nu \int_{0}^{t} k^{2}+|\eta-k(\tau-t)|^{2} d \tau\right] \\
& =\frac{1}{\left(k^{2}+\eta^{2}\right)\langle\eta+k t\rangle^{2}}\langle\eta+k t\rangle^{2} \widehat{\omega}_{\text {in }}(k, \eta+k t) \exp \left[-\nu \int_{0}^{t} k^{2}+|\eta-k(\tau-t)|^{2} d \tau\right],
\end{aligned}
$$

which implies

$$
\left\|\psi_{\neq}(t)\right\|_{L^{2}} \lesssim \frac{1}{\langle t\rangle^{2}}\left\|\omega_{\text {in }}\right\|_{H^{2}} e^{-c \nu t^{3}} .
$$


In particular, it follows that there is some decay which is independent of Reynolds number, and indeed is present even in the case $\nu=0$. Moreover, by similar arguments, one has

$$
\left\|\partial_{x} \psi_{\neq}(t)\right\|_{L^{2}}+\langle t\rangle^{-1}\left\|\partial_{y} \psi_{\neq}(t)\right\|_{L^{2}} \lesssim \frac{1}{\langle t\rangle^{2}}\left\|\omega_{\text {in }}\right\|_{H^{2}} e^{-c \nu t^{3}}
$$

which implies that the velocity field converges back to a shear flow even at infinite Reynolds number. The name inviscid damping is due to its relationship with Landau damping in plasma physics (see [LZ11, Zil14, BM13, WZZ15] and the references therein for more discussion on inviscid damping and [CM98, Ryu99, MV11, BMM13] for discussions regarding Landau damping). The regularity loss in (1.9) is physically meaningful and in particular is connected to a transient (non-normal) unmixing effect known as the Orr mechanism. When $\gamma$ is too small, this loss makes it impossible to naively close the kind of regularity estimates required to apply (1.8) due to the large derivative loss in the right hand side (see [BM13] for a more in-depth discussion). Physically, this manifests as the nonlinear echo resonance [YD02, YDO05, MV11, BM13]. In this paper we are able to balance this difficulty with the enhanced dissipation. This balance is what sets the requirement $\varepsilon \ll \nu^{1 / 2}$ used here (see e.g. the treatment of estimate (2.13) below).

1.3. Notations used in this paper. Throughout this paper, we write

$$
\langle x\rangle=\sqrt{1+x^{2}} \text {. }
$$

For a function $h(x, y)$, denote by

$$
h_{0}(y)=\int_{\mathbb{T}} h(x, y) d x \quad \text { and } \quad h_{\neq}(x, y)=h(x, y)-h_{0}(y),
$$

the projections onto 0 frequencies with respect to $x$ and the projection onto non-zero frequencies, respectively. By convention, we use $k, l$ to represent the Fourier variable of $x$, while $\xi, \eta$ are the Fourier variable of $y$. The Fourier transform of a function $a$ is denoted by $\hat{a}(k, \xi)$. We use the notation $f \lesssim g$ to express $f \leq C g$ for some constant $C>0$ that is independent of the parameters of interest and use $f \ll g$ when $f \leq \varepsilon_{0} g$ for some universal constant $\varepsilon_{0}$ sufficiently close to 0 . The notation $L^{p} L^{q}=L_{t}^{p} L_{x, y}^{q}$ is used for the Banach space $L^{p}\left([0, T] ; L^{q}(\Omega)\right)$ with norm $\|f(t, x)\|_{L^{p} L^{q}}^{p}=\int_{0}^{T}\left(\int_{\Omega}|f|^{q} d x\right)^{p / q} d t$ where $p, q \in[1, \infty]$ and $T>0$. We also use the space $L^{p} H^{s}=L_{t}^{p} H_{x, y}^{s}$ whose norm is given $\|f\|_{L^{p} H^{s}}^{p}=\int_{0}^{T}\|f\|_{H^{s}}^{p} d t$ for $p \in[1, \infty]$ and $s \geq 0$.

\section{Stability threshold for the Couette flow}

In order to introduce several of the main ideas of the proof, we first prove Theorem 1.1 in the case that $U(y)=\bar{U}(t, y)=y$. We change coordinates to mod out by the fast mixing of the Couette flow, however, unlike the previous works on Navier-Stokes and Euler [BM13, BMV16, BGM15a, BGM15b, BGM15c], we do not need a coordinate change that depends on the solution itself:

$$
\begin{aligned}
& z=x-t v \\
& v=y .
\end{aligned}
$$

The change $v \mapsto y$ is made only to unify with Section 3 below. If we then write $f(t, z, v)=$ $\omega(t, z+t v, v)$, the Navier-Stokes equations (1.4) become

$$
\begin{aligned}
& \partial_{t} f+u \cdot \nabla_{L} f=\nu \Delta_{L} f \\
& u=-\nabla_{L}^{\perp}\left(-\Delta_{L}\right)^{-1} f,
\end{aligned}
$$


where

$$
\begin{aligned}
& \nabla_{L}=\left(\begin{array}{c}
\partial_{z} \\
\partial_{v}-t \partial_{z}
\end{array}\right) \\
& \Delta_{L}=\nabla_{L} \cdot \nabla_{L}=\partial_{z z}+\left(\partial_{v}-t \partial_{z}\right)^{2} .
\end{aligned}
$$

The following statement in the new coordinates then implies Theorem 1.1 (in the case $U(y)=y$ ).

THEOREM 2.1. Let $N>1$ and assume $\left\|f_{\text {in }}\right\|_{H^{N}}=\varepsilon \ll \nu^{1 / 2}$ with $0<\nu \leq 1$. Then the unique global in time solution $f$ to $(2.2 \mathrm{a})-(2.2 \mathrm{~b})$ is such that

$$
\|f\|_{L^{\infty} H^{N}}+\nu^{1 / 2}\left\|\nabla_{L} f\right\|_{L^{2} H^{N}} \lesssim \varepsilon
$$

and

$$
\left\|f_{\neq}\right\|_{L^{2} H^{N}} \lesssim \varepsilon \nu^{-1 / 6}
$$

where the implicit constants do not depend on $\nu$ or on the initial datum.

The norm we employ is based on a special, time-dependent Fourier multiplier which is designed to capture transient unmixing effects in the linearization and its effect on the nonlinear problem. This particular technique was first introduced in [BM13], although the norm we use is more similar to the 3D Sobolev regularity work [BGM15c]. We specifically employ a Fourier multiplier $M$ which obeys the properties:

$$
\begin{aligned}
M(0, k, \xi) & =M(t, 0, \xi)=1 \\
1 & \geq M(t, k, \xi) \geq c \\
-\frac{\dot{M}}{M} & \geq \frac{|k|}{k^{2}+|\xi-k t|^{2}} \quad \text { for } k \neq 0 \\
\left|\frac{\partial_{\xi} M(k, \xi)}{M(k, \xi)}\right| & \lesssim \frac{1}{|k|} \quad \text { for } k \neq 0, \text { uniformly in } \xi \\
1 & \lesssim \nu^{-1 / 6}\left(\sqrt{-\dot{M} M(t, k, \eta)}+\nu^{1 / 2}|k, \eta-k t|\right) \quad \text { for } k \neq 0 \\
\sqrt{-\dot{M} M(t, k, \eta)} & \lesssim\langle\eta-\xi\rangle \sqrt{-\dot{M} M(t, k, \xi)},
\end{aligned}
$$

for a constant $c \in(0,1)$ which is independent of $\nu$. The construction of such a multiplier $M$ is given in Appendix A below and is similar to one used in [BGM15c] (some aspects of the multiplier also appeared in [Zil14]). See Lemma A.1 in Appendix A for details.

Proof of Theorem 2.1. Defining

$$
A=M\langle D\rangle^{N}
$$

where $\langle D\rangle=\sqrt{1+D^{2}}$, it follows from Plancherel's inequality and (2.6b) that

$$
\|f\|_{H^{N}} \lesssim c\|A(t) f\|_{L^{2}} \leq\|f\|_{H^{N}}
$$

and that $\|A(0) f\|_{2}=\|f\|_{H^{N}}$. It is important to note that such a simple equivalence to a standard norm (uniform in $\nu$ and $t$ ) has not been true in most previous works using similar Fourier multiplierbased techniques [BM13, BMV16, BGM15a, BGM15b, BGM15c]; the exception being [Zil14]. This distinction is one of the primary reasons that the proof of Theorem 1.1 is significantly less technical than the previous works (it also makes the technique essentially a Fourier-side analogue of Alinhac's ghost energy method [Ali01]; see [BGM15c] for more discussion). 
By (2.7), the proof of Theorem 2.1 is then based on establishing the a priori estimate

$$
\|A f\|_{L^{\infty} L^{2}}+\nu^{1 / 2}\left\|\nabla_{L} A f\right\|_{L^{2} L^{2}}+\left\|\sqrt{-\dot{M} M}\langle D\rangle^{N} f\right\|_{L^{2} L^{2}} \leq 8 \varepsilon
$$

In turn, (2.8) implies (2.4) since $\|A f\|_{L^{2}}$ and $\|f\|_{H^{N}}$ are equivalent. Moreover, (2.5) follows from (2.8), since (2.6e) and (2.7) imply that for any $f$,

$$
\left\|f_{\neq}\right\|_{L^{2} H^{N}} \lesssim \nu^{-1 / 6}\left(\nu^{1 / 2}\left\|\nabla_{L} A f_{\neq}\right\|_{L^{2} L^{2}}+\left\|\sqrt{-\dot{M} M}\langle D\rangle^{N} f\right\|_{L^{2} L^{2}}\right) .
$$

By the local well-posedness in $H^{N}$ of the 2D Navier-Stokes equations, for $t$ sufficiently small, there holds

$$
\|A f\|_{L^{\infty}\left(0, t ; L^{2}\right)}+\nu^{1 / 2}\left\|\nabla_{L} A f\right\|_{L^{2}\left(0, t ; L^{2}\right)}+\left\|\sqrt{-\dot{M} M}\langle D\rangle^{N} f\right\|_{L^{2}\left(0, t ; L^{2}\right)}<2 \varepsilon,
$$

and all the quantities on the right hand side of (2.8) take values continuously in time. We will use a bootstrap argument to extend these estimates for all time. Hence, define $T \leq \infty$ to be the maximal time such that

$$
\|A f\|_{L^{\infty}\left(0, T ; L^{2}\right)}+\nu^{1 / 2}\left\|\nabla_{L} A f\right\|_{L^{2}\left(0, T ; L^{2}\right)}+\left\|\sqrt{-\dot{M} M}\langle D\rangle^{N} f\right\|_{L^{2}\left(0, T ; L^{2}\right)} \leq 8 \varepsilon
$$

holds; by the above discussion, $T>t$. We refer to this inequality as the bootstrap assumption. We next prove that in fact (2.10) holds with the constant 8 on the right side replaced by 4 , implying the global stability (i.e. that $T$ may be taken arbitrarily large).

Proposition 2.2. For all $N>1$ and $\varepsilon$ sufficiently small (depending only on $N$ ), inequality (2.10) holds with the " 8 " replaced with a " 4 " on $[0, T)$, and hence by continuity, $T=+\infty$.

First, applying the operator $A$ to (2.2a), we obtain the energy estimate for $f$ which reads

$$
\begin{gathered}
\frac{1}{2} \frac{d}{d t}\|A f(t)\|_{L^{2}}^{2}+\nu\left\|\nabla_{L} A f(t)\right\|_{L^{2}}^{2}+\left\|\sqrt{-\dot{M} M}\langle D\rangle^{N} f(t)\right\|_{L^{2}}^{2} \\
=-\int A\left(u(t) \cdot \nabla_{L} f(t)\right) A f(t) d V
\end{gathered}
$$

where we use the notation $d V=d z d v$. Integrating the above equality in time on the interval [0, T], we obtain (where we have abbreviated the time-interval from norms in order to reduce clutter)

$$
\begin{aligned}
& \frac{1}{2}\|A f(T)\|_{L^{2}}^{2}+\nu\left\|\nabla_{L} A f\right\|_{L^{2} L^{2}}^{2}+\left\|\sqrt{-\dot{M} M}\langle D\rangle^{N} f\right\|_{L^{2} L^{2}}^{2} \\
& \quad=\frac{1}{2}\|A f(0)\|_{L^{2}}^{2}-\iint A\left(u \cdot \nabla_{L} f\right) A f d V d t=\frac{1}{2}\|A f(0)\|_{L^{2}}^{2}-\mathcal{T}
\end{aligned}
$$

where $\mathcal{T}=\iint A\left(u \cdot \nabla_{L} f\right) A f d V d t$. In order to estimate $\mathcal{T}$, which is the bulk of the proof, we decompose the velocity $u$ into the zero frequency and non-zero frequency parts as

$$
\mathcal{T}=\iint A\left(u_{0}^{x} \partial_{z} f\right) A f d V d t+\iint A\left(u_{\neq} \cdot \nabla_{L} f\right) A f d V d t=\mathcal{T}_{0}+\mathcal{T}_{\neq} .
$$

We first bound the term $\mathcal{T}_{\neq}$using (2.6b) and the algebra property of $H^{N}$ as

$$
\begin{aligned}
\left|\mathcal{T}_{\neq}\right| & =\left|\iint A\left(\nabla_{L}^{\perp} \Delta_{L}^{-1} f_{\neq} \cdot \nabla_{L} f\right) A f d V d t\right| \\
& \lesssim\left\|\nabla_{L}^{\perp} \Delta_{L}^{-1} f_{\neq}\right\|_{L^{2} H^{N}}\left\|\nabla_{L} f\right\|_{L^{2} H^{N}}\|A f\|_{L^{\infty} L^{2} .}
\end{aligned}
$$


From (2.12), the property (2.6c) of $M$, and the fact that $f_{\neq}$contains only modes $|k| \geq 1$, we obtain the following, using the bootstrap assumption (2.10),

$$
\begin{aligned}
\left|\mathcal{T}_{\neq}\right| & \lesssim\left\|\sqrt{-\dot{M} M}\langle D\rangle^{N} f_{\neq}\right\|_{L^{2} L^{2}}\left\|\nabla_{L} f\right\|_{L^{2} H^{N}}\|A f\|_{L^{\infty} L^{2}} \\
& \lesssim \varepsilon^{3} \nu^{-1 / 2} .
\end{aligned}
$$

This is consistent with Proposition 2.2 by choosing $\varepsilon \ll \nu^{1 / 2}$ (where the implicit constant depends only on $N$ ). This term, $\mathcal{T}_{\neq}$, is the only critical one for which we need the assumption $\varepsilon \ll \nu^{1 / 2}$.

In order to estimate the zero frequency part, we note that $f_{0}$ is independent of $z$ and thus we may rewrite $\mathcal{T}_{0}$ as

$$
\mathcal{T}_{0}=\iint A\left(-\partial_{v} \partial_{v}^{-2} f_{0} \partial_{z} f\right) A f d V d t=\iint A\left(-\partial_{v} \partial_{v}^{-2} f_{0} \partial_{z} f_{\neq}\right) A f_{\neq} d V d t .
$$

where we also used, by Plancherel's theorem,

$$
\int A\left(-\partial_{v} \partial_{v}^{-2} f_{0} \partial_{z} f_{\neq}\right) A f_{0} d V=0
$$

Additionally, using the cancellation

$$
\int \partial_{v} \partial_{v}^{-2} f_{0} \partial_{z} A f_{\neq} A f_{\neq} d V=0
$$

we obtain from (2.14) that

$$
\mathcal{T}_{0}=\iint\left(A\left(-\partial_{v} \partial_{v}^{-2} f_{0} \partial_{z} f_{\neq}\right)+\partial_{v} \partial_{v}^{-2} f_{0} \partial_{z} A f_{\neq}\right) A f_{\neq} d V d t
$$

By Plancherel's theorem,

$$
-\mathcal{T}_{0}=\frac{1}{4 \pi^{2}} \sum_{k \neq 0} \iiint(A(k, \xi)-A(k, \xi-\eta)) \frac{\eta}{\eta^{2}} \hat{f}(0, \eta) k \hat{f}(k, \xi-\eta) A(k, \xi) \overline{\hat{f}}(k, \xi) d \eta d \xi d t .
$$

We decompose the difference (a commutator in real variables) $A(k, \xi)-A(k, \xi-\eta)$ into two commutators, one due to $M$ and one due to $\langle D\rangle^{N}$ :

$$
\begin{aligned}
A(k, \xi)-A(k, \xi-\eta)= & M(k, \xi)\left(\left(1+k^{2}+\xi^{2}\right)^{N / 2}-\left(1+k^{2}+(\xi-\eta)^{2}\right)^{N / 2}\right) \\
& +(M(k, \xi)-M(k, \xi-\eta))\left(1+k^{2}+(\xi-\eta)^{2}\right)^{N / 2} \\
= & \operatorname{com}_{1}+\operatorname{com}_{2} .
\end{aligned}
$$

For $\operatorname{com}_{1}$, we use the mean value theorem to obtain, for some $\theta \in[0,1]$,

$$
\begin{aligned}
\left|\operatorname{com}_{1}\right| & \left.=\mid M(k, \xi) \frac{N}{2}\left(1+k^{2}+(\xi-\theta \eta)^{2}\right)^{N / 2-1}\right) 2(\xi-\theta \eta) \eta \mid \\
& \lesssim\left(\left(1+k^{2}+(\xi-\eta)^{2}\right)^{(N-1) / 2}+\left(1+k^{2}+\xi^{2}\right)^{(N-1) / 2}\right)|\eta| .
\end{aligned}
$$

To estimate $\mathrm{com}_{2}$, we additionally recall that the multiplier $M$ satisfies (2.6d). From (2.6d) and the mean value theorem we deduce

$$
\left|\operatorname{com}_{2}\right| \lesssim \frac{|\eta|}{|k|}\left(1+k^{2}+(\xi-\eta)^{2}\right)^{N / 2}
$$


Combining (2.16) with the bounds (2.17) and (2.18) for $\mathrm{com}_{1}$ and $\mathrm{com}_{2}$ respectively, we arrive at

$$
\begin{aligned}
\left|\mathcal{T}_{0}\right| \lesssim \sum_{k \neq 0} \iint & \int\left(\left(1+k^{2}+(\xi-\eta)^{2}\right)^{N / 2}+\left(1+k^{2}+\xi^{2}\right)^{N / 2}\right) \\
& \times|\hat{f}(0, \eta) \hat{f}(k, \xi-\eta) \| A(k, \xi) \hat{\hat{f}}(k, \xi)| d \eta d \xi d t
\end{aligned}
$$

By Young's convolution inequality, and $N>1$, we obtain,

$$
\left|\mathcal{T}_{0}\right| \lesssim\left\|f_{0}\right\|_{L^{\infty} H^{N}}\left\|f_{\neq}\right\|_{L^{2} H^{N}}^{2}
$$

By (2.9) and the bootstrap assumption (2.10), we obtain

$$
\begin{aligned}
\left|\mathcal{T}_{0}\right| & \lesssim\left\|f_{0}\right\|_{L^{\infty} H^{N}} \nu^{-1 / 3}\left(\left\|\sqrt{-\dot{M} M}\langle D\rangle^{N} f_{\neq}\right\|_{L^{2} L^{2}}+\nu^{1 / 2}\left\|\nabla_{L} A f_{\neq}\right\|_{L^{2} L^{2}}\right)^{2} \\
& \lesssim \varepsilon^{3} \nu^{-1 / 3},
\end{aligned}
$$

which is sufficient to deduce Proposition 2.2 under the hypotheses $\varepsilon \ll \nu^{-1 / 3}$. This completes the proof of Proposition 2.2 and hence of Theorem 2.1.

\section{Shear flows close to Couette}

In this section we consider the more general class of shear flows with initial data $(U(y), 0)$ which are sufficiently close to Couette, in the sense that

$$
\left\|U^{\prime}-1\right\|_{H^{s}}+\left\|U^{\prime \prime}\right\|_{H^{s}} \leq \delta \ll 1
$$

for some $s \geq 2+N$.

REMARK 3.1. Recall from Theorem 1.1 that the constant $\delta$ is independent of $\varepsilon$ and $\nu$.

Recall the definition of $\bar{U}$ from (1.2), the solution of the Navier-Stokes equations with the initial datum $(U(y), 0)$. From standard estimates on the heat equation there holds,

$$
\begin{aligned}
\sup _{t>0}\left\|\bar{U}^{\prime}(t, \cdot)-1\right\|_{H^{s}} & \leq\left\|U^{\prime}-1\right\|_{H^{s}} \\
\sup _{t>0}\left\|\bar{U}^{\prime \prime}(t, \cdot)\right\|_{H^{s}} & \leq\left\|U^{\prime \prime}\right\|_{H^{s}} \\
\left\|\bar{U}^{\prime \prime}\right\|_{L_{t}^{2} H_{y}^{s}} & \lesssim \delta \nu^{-1 / 2} .
\end{aligned}
$$

3.1. Coordinate System. Recall that $\bar{U}(t)=e^{\nu t \partial_{y y}} U$ is the decaying background shear flow. Instead of (2.1) we need to work with a coordinate change adapted to this shear flow, and thus consider

$$
\begin{aligned}
x \mapsto z & =x-t \bar{U}(t, y) \\
y \mapsto v & =\bar{U}(t, y) .
\end{aligned}
$$

For $\delta$ sufficiently small, the map $(x, y) \mapsto(x, v)$ is invertible; see Subsection 3.2 below. The choice $y \mapsto v$ is made so that $\partial_{y} \mapsto \partial_{y} \bar{U}\left(\partial_{v}-t \partial_{z}\right)$ (see (3.6) below). This ensures that the critical times are not significantly perturbed by the coefficients, and featured in all of the previous works on Navier-Stokes or Euler near Couette flow, introduced first in [BM13]. The change from $x \mapsto z$ is essentially rewinding by the characteristics of the shear flow, although in way which is also well-adjusted for the requirement on $v$ (it is also well-adjusted for the parabolic decay of $\bar{U}(t, y)$, although this is less obvious). 
In the new coordinate system we define quantities corresponding to vorticity, stream function, and velocity, respectively, by

$$
\begin{aligned}
& f(t, z(t, x, y), v(t, y))=\omega(t, x, y) \\
& \phi(t, z(t, x, y), v(t, y))=\psi(t, x, y) \\
& u(t, z(t, x, y), v(t, y))=\bar{v}(t, x, y) .
\end{aligned}
$$

It is also convenient to denote the spatial derivatives of the shear flow in the new coordinates

$$
\begin{aligned}
& a(t, v(t, y))=\bar{U}^{\prime}(t, y) \\
& b(t, v(t, y))=\bar{U}^{\prime \prime}(t, y) .
\end{aligned}
$$

For any function $\widetilde{h}$ in the $(x, y)$ coordinates, the corresponding function $h$ in the $(z, v)$ coordinates

$$
h(t, z, v)=\widetilde{h}(t, x, y),
$$

the differential operator $\nabla \mapsto \nabla_{t}$, is defined by

$$
\nabla \widetilde{h}(t, x, y)=\left(\begin{array}{c}
\partial_{x} \widetilde{h} \\
\partial_{y} \widetilde{h}
\end{array}\right)=\left(\begin{array}{c}
\partial_{z} h \\
a\left(\partial_{v}-t \partial_{z}\right) h
\end{array}\right)=\left(\begin{array}{c}
\partial_{z}^{t} h \\
\partial_{v}^{t} h
\end{array}\right)=\nabla_{t} h,
$$

and analogously $\Delta \mapsto \Delta_{t}$ is defined via,

$$
\Delta \widetilde{h}(t, x, y)=\left(\partial_{z}^{2}+a^{2} \partial_{v v}^{L}+b \partial_{v}^{L}\right) h=\Delta_{t} h .
$$

Finally, the time derivative maps to the following, using $\partial_{t} \bar{U}=\nu \partial_{y y} \bar{U}$ and the definitions of $a, b$ from (3.5),

$$
\begin{aligned}
\partial_{t} \widetilde{h} & =\partial_{t} h+\partial_{z} h\left(-\bar{U}-t \partial_{t} \bar{U}\right)+\partial_{v} h \partial_{t} \bar{U} \\
& =\partial_{t} h-v \partial_{z} h+\nu b\left(\partial_{v}-t \partial_{z}\right) h .
\end{aligned}
$$

Recall that (2.3) is the "linear part" of the operator $\nabla_{t}$. The relationship between $f$ and $\phi$ is given through (3.7):

$$
\begin{aligned}
f & =\Delta_{t} \phi=\left(\partial_{z}^{2}+a^{2} \partial_{v v}^{L}+b \partial_{v}^{L}\right) \phi=\left(\partial_{z}^{2}+a^{2}\left(\partial_{v}-t \partial_{z}\right)^{2}+b\left(\partial_{v}-t \partial_{z}\right)\right) \phi \\
& =\Delta_{L} \phi+\left(\left(a^{2}-1\right) \partial_{v v}^{L}+b \partial_{v}^{L}\right) \phi .
\end{aligned}
$$

Note that by the chain rule, we have

$$
b=a \partial_{v} a .
$$

Using the above notations, by applying (2.3), (3.7), and (3.8) we find that $f$ satisfies the equations

$$
\begin{aligned}
& \partial_{t} f+u \cdot \nabla_{t} f=b \partial_{z} \phi+\nu \widetilde{\Delta}_{t} f \\
& \Delta_{t} \phi=f \\
& u=\nabla_{t}^{\perp} \phi,
\end{aligned}
$$

where the modified Laplace operator is given by

$$
\widetilde{\Delta}_{t}=\partial_{z}^{2}+a^{2} \partial_{v v}^{L}=\Delta_{L}+\left(a^{2}-1\right) \partial_{v v}^{L} .
$$

In particular, note the cancellation between the lower order term in $\Delta_{t}$ and the last term in (3.8).

REMARK 3.2. Recall the notations in (1.10). As $\bar{U}$ is independent of $x$, there holds

$$
\left(\nabla_{t} h\right)_{0}=\nabla_{t} h_{0}, \quad\left(\nabla_{t} h\right)_{\neq}=\nabla_{t} h_{\neq} .
$$

As a result, from the divergence free condition we deduce that $u_{0}^{y}=0$. 
3.2. Equivalence of the two coordinate systems. We shall prove Theorem 1.1 in the new coordinate system (3.3). Here we discuss how to relate this coordinate system to the original $(x, y)$. The first lemma, the proof of which can be found in [IKT13], provides a composition inequality in fractional Sobolev spaces.

Lemma 3.3 (Fractional Sobolev Composition). Let $s^{\prime}>2, s^{\prime} \geq s \geq 0, f \in H^{s}(\mathbb{R})$, and $g \in H^{s^{\prime}}(\mathbb{R})$ be such that $\|g\|_{H^{s^{\prime}}} \leq \delta$. Then, there holds

$$
\|f \circ(I+g)\|_{H^{s}} \leq C_{s, s^{\prime}}(\delta)\|f\|_{H^{s}}
$$

where the implicit constant obeys $C_{s, s^{\prime}}(\delta) \rightarrow 1$ as $\delta \rightarrow 0$.

The next implicit function theorem is important for inverting the coordinate transformation (3.3) and carrying the information back to the original coordinates.

Lemma 3.4 (Implicit Function Theorem). Let $s>2$. There exists an $\varepsilon_{0}=\varepsilon_{0}(s)$ such that if $\|\alpha\|_{H^{s}} \leq \varepsilon_{0}$, then there is a unique solution $\beta$ to

$$
\beta(y)=\alpha(y+\beta(y))
$$

with $\|\beta\|_{H^{s}} \lesssim \varepsilon_{0}$.

The next lemma shows that we are allowed to take all the information in the original system to the new system.

Lemma 3.5. Let $s^{\prime}>2, s^{\prime} \geq s \geq 0, f \in H^{s}\left(\mathbb{R}^{2}\right)$, and $g \in H^{s^{\prime}}\left(\mathbb{R}^{2}\right)$ be such that $\|g\|_{H^{s^{\prime}}} \leq \delta$. Then, there holds

$$
\|f\|_{H^{s}} \leq C_{s, s^{\prime}}(\delta)\|f \circ(I+g)\|_{H^{s}}
$$

where the implicit constant obeys $C_{s, s^{\prime}}(\delta) \rightarrow 1$ as $\delta \rightarrow 0$.

Proof of Lemma 3.5. Let $h=(I+g)(y)=y+g(y)$. Then we rewrite this equality as $h-y=g(h-y+h)$. Now thinking of $y$ as a function of $h$, we denote $\beta(h)=h-y$ and deduce $\beta(h)=g(h+\beta(h))$. By Lemma 3.4, there exists a $\beta$ such that the above equality holds and furthermore

$$
\|\beta\|_{H^{s}} \lesssim \delta
$$

Now we write $f$ as

$$
f(h)=f \circ(I+g) \circ(I+\beta)(h)
$$

and use Lemma 3.3 to conclude the proof.

From the properties of the heat equation and Lemma 3.5, we can deduce the following lemma regarding the coefficients $a$ and $b$, which arise in (3.11).

LEMma 3.6. From (3.10), Lemma 3.5, and (3.2c), for $\delta$ sufficiently small, there holds for $\sigma>2$,

$$
\left\|\partial_{v}\left(a^{2}-1\right)\right\|_{L_{t}^{2} H_{y}^{\sigma}}=\|2 b\|_{L_{t}^{2} H_{y}^{\sigma}} \lesssim\left\|\bar{U}^{\prime \prime}\right\|_{L_{t}^{2} H_{y}^{\sigma}} \lesssim \delta \nu^{-1 / 2},
$$

where the implicit constant does not depend on $\delta$ and $\nu$. Similarly, by (3.4), (3.1), and Lemma 3.5, we have that

$$
\|a-1\|_{H^{\sigma}}+\|b\|_{H^{\sigma}} \leq 2\left(\left\|\bar{U}^{\prime}-1\right\|_{H^{\sigma}}+\left\|\bar{U}^{\prime \prime}\right\|_{H^{\sigma}}\right) \leq 2 \delta
$$

holds for $\sigma>2$ and $\delta>0$ small enough. 
3.3. Main result. The main result of this section, which in particular implies Theorem 1.1, is:

THEOREM 3.7. Let $N>1$ and assume that the shear flow $(U(y), 0)$ satisfies (3.1). If the initial vorticity perturbation obeys $\left\|f_{\text {in }}\right\|_{H^{N}}=\varepsilon \ll \nu^{1 / 2}$, then the unique solution $f$ to (3.11a)-(3.11c) obeys the global in time estimates

$$
\begin{aligned}
& \|f\|_{L^{\infty} H^{N}}+\nu^{1 / 2}\left\|\nabla_{L} f\right\|_{L^{2} H^{N}} \lesssim \varepsilon \\
& \left\|u_{0}^{x}\right\|_{L^{\infty} L^{2}}+\nu^{1 / 2}\left\|\partial_{v} u_{0}^{x}\right\|_{L^{2} L^{2}} \lesssim \varepsilon
\end{aligned}
$$

and

$$
\left\|f_{\neq}\right\|_{L^{2} H^{N}} \lesssim \varepsilon \nu^{-1 / 6}
$$

where the implicit constants do not depend on $\nu$ or on the initial datum.

Indeed, the estimates (3.14) and (3.15) together with Lemmas 3.3 and 3.5, imply Theorem 1.1.

3.4. Proof of Theorem 3.7. The proof of Theorem 3.7 uses the main ideas of Theorem 2.1, however, due to the more complicated change of coordinates (3.3), we need to consider new terms, such as the linear term $b \partial_{z} \phi$ appearing in (3.11a). Moreover, due to (3.3), the Biot-Savart law (3.11b)-(3.11c) is more complicated, and in particular, (3.11c) is no longer expressible as a Fourier multiplier (this was possible in the proof of Theorem 2.1), which in turn makes the use of our norm $A$ a little more difficult.

Proof of TheOrem 3.7. For our norm, we use the same multipliers $M$ and $A$ as in the proof of Theorem 2.1 (the $M$ which obeys the conditions (2.6)). Let $T$ be the maximal time interval $[0, T]$ such that the following estimates hold:

$$
\begin{aligned}
& \|A f\|_{L^{\infty} L^{2}}+\nu^{1 / 2}\left\|\nabla_{L} A f\right\|_{L^{2} L^{2}}+\left\|\sqrt{-\dot{M} M}\langle D\rangle^{N} f\right\|_{L^{2} L^{2}} \leq 8 \varepsilon \\
& \left\|u_{0}^{z}\right\|_{L^{\infty} L^{2}}+\nu^{1 / 2}\left\|\partial_{v} u_{0}^{z}\right\|_{L^{2} L^{2}} \leq 8 \varepsilon .
\end{aligned}
$$

By local well-posedness, the quantities on the left-hand side of (3.16) take values continuously in time, $T>0$, and (3.16) holds on a smaller time interval with the " 8 " replaced by a " 2 ". The following proposition implies Theorem 3.7.

PROPOSITION 3.8. For $\delta$ and $\varepsilon$ chosen sufficiently small, the estimates in (3.16) hold on $[0, T]$ with " 8 " replaced with " 4 ". It follows by continuity that $T=+\infty$.

We now prove Proposition 3.8.

3.4.1. Energy estimate on $f$, (3.16a). In this subsection we improve (3.16a). Applying the operator $A$ to (3.11a) we arrive at the energy estimate for the vorticity $f$ :

$$
\begin{aligned}
\frac{1}{2}\|A f(T)\|_{L^{2}}^{2}+\nu\left\|\nabla_{L} A f(T)\right\|_{L^{2} L^{2}}^{2}+\left\|\sqrt{-\dot{M} M}\langle D\rangle^{N} f(T)\right\|_{L^{2} L^{2}}^{2} \\
=\frac{1}{2}\|A f(0)\|_{L^{2}}^{2}-\iint A\left(u \cdot \nabla_{t} f\right) A f d V d t+\iint A\left(b \partial_{z} \phi\right) A f d V d t \\
\quad+\nu \iint A\left(\left(a^{2}-1\right) \partial_{v v}^{L} f\right) A f d V d t \\
=\frac{1}{2}\|A f(0)\|_{L^{2}}^{2}-\mathcal{T}+\mathrm{S}+\mathrm{DE} .
\end{aligned}
$$

We next bound the transport, the source, and the dissipation error terms on the right side of (3.17). 
We first consider the transport term $\mathcal{T}$. We begin by decomposing the velocity field into zero and non-zero modes (recalling (3.11b)-(3.11c), and the definition of $\nabla_{t}$ ), we have

$$
\begin{aligned}
\mathcal{T} & =\iint A\left(u_{0}^{z} \partial_{z} f\right) A f d V d t+\iint A\left(\nabla_{t}^{\perp} \Delta_{t}^{-1} f_{\neq} \cdot \nabla_{t} f\right) A f d V d t \\
& =\mathcal{T}_{0}+\mathcal{T}_{\neq} .
\end{aligned}
$$

Using (3.13) and the $H^{N}$ product rule $(N>1)$, the term $\mathcal{T}_{\neq}$is bounded as

$$
\begin{aligned}
\left|\mathcal{T}_{\neq}\right| & \lesssim\left\|\nabla_{t}^{\perp} \Delta_{t}^{-1} f_{\neq}\right\|_{L^{2} H^{N}}\left\|\nabla_{t} f\right\|_{L^{2} H^{N}}\|A f\|_{L^{\infty} L^{2}} \\
& \lesssim\left\|\nabla_{L}^{\perp} \Delta_{t}^{-1} f_{\neq}\right\|_{L^{2} H^{N}}\left\|\nabla_{L} f\right\|_{L^{2} H^{N}}\|A f\|_{L^{\infty} L^{2}}
\end{aligned}
$$

By using (2.6c), the fact that $|k| \geq 1$, and the Lemma (A.3), which allows us to commute $\Delta_{t}^{-1}$ and $\sqrt{-\dot{M} M}$, there holds

$$
\left\|\nabla_{L}^{\perp} \Delta_{t}^{-1} f_{\neq}\right\|_{L^{2} H^{N}} \lesssim\left\|\sqrt{-\dot{M} M} \Delta_{L} \Delta_{t}^{-1} f_{\neq}\right\|_{L^{2} H^{N}} \lesssim\left\|\sqrt{-\dot{M} M} f_{\neq}\right\|_{L^{2} H^{N}} .
$$

From (3.18) and (3.16), we then obtain

$$
\left|\mathcal{T}_{\neq}\right| \lesssim\left\|\sqrt{-\dot{M} M} f_{\neq}\right\|_{L^{2} H^{N}}\left\|\nabla_{L} f\right\|_{L^{2} H^{N}}\|A f\|_{L^{\infty} L^{2}} \lesssim \varepsilon^{3} \nu^{-1 / 2}
$$

This is consistent with Proposition 3.8 provided we use the hypothesis that $\varepsilon \ll \nu^{1 / 2}$.

For the zero mode term $\mathcal{T}_{0}$, we begin as in Section 2. Similarly to (2.15), upon integrating by parts we need to consider the commutator

$$
\mathcal{T}_{0}=-\iint\left(A\left(u_{0}^{z} \partial_{z} f_{\neq}\right)-u_{0}^{z} \partial_{z} A f_{\neq}\right) A f_{\neq} d V d t .
$$

By Plancherel's theorem,

$$
-\mathcal{T}_{0}=\frac{1}{4 \pi^{2}} \sum_{k \neq 0} \int_{t} \int_{\xi} \int_{\eta}(A(k, \xi)-A(k, \xi-\eta)) \widehat{u_{0}^{z}}(\eta) i k \hat{f}(k, \xi-\eta) A(k, \xi) \overline{\hat{f}}(k, \xi) d \eta d \xi d t .
$$

The commutator is estimated as in (2.19) above (in particular, we apply (2.17) and (2.18)); we also apply $u_{0}^{z}=a \partial_{v} \Delta_{t}^{-1} f_{0}$,

$$
\begin{aligned}
\left|\mathcal{T}_{0}\right| & \lesssim\left\|\partial_{v}\left(a \partial_{v} \Delta_{t}^{-1} f_{0}\right)\right\|_{L^{\infty} H^{N}}\left\|f_{\neq}\right\|_{L^{2} H^{N}}\left\|A f_{\neq}\right\|_{L^{2} L^{2}} \\
& \lesssim\left(\|a-1\|_{L^{\infty} H^{N+1}}\left\|\partial_{v} \Delta_{t}^{-1} f_{0}\right\|_{L^{\infty} H^{N}}+\left\|a \partial_{v v} \Delta_{t}^{-1} f_{0}\right\|_{L^{\infty} H^{N}}\right)\left\|f_{\neq}\right\|_{L^{2} H^{N}}\left\|A f_{\neq}\right\|_{L^{2} L^{2}} .
\end{aligned}
$$

Note that by considering separately frequencies less than and greater than one, there holds

$$
\left\|\partial_{v} \Delta_{t}^{-1} f_{0}\right\|_{L^{\infty} H^{N}} \leq\left\|\partial_{v} \Delta_{t}^{-1} f_{0}\right\|_{L^{\infty} L^{2}}+\left\|\partial_{v v} \Delta_{t}^{-1} f_{0}\right\|_{L^{\infty} H^{N}}
$$

Therefore, by (3.13), (2.9), Lemma A.5, and Lemma 3.5 (see also (3.20) below), we further obtain

$$
\begin{aligned}
\left|\mathcal{T}_{0}\right| & \lesssim\left(\left\|\partial_{v} \Delta_{t}^{-1} f_{0}\right\|_{L^{\infty} L^{2}}+\left\|\partial_{v v} \Delta_{t}^{-1} f_{0}\right\|_{L^{\infty} H^{N}}\right)\left\|f_{\neq}\right\|_{L^{2} H^{N}}\left\|A f_{\neq}\right\|_{L^{2} L^{2}} \\
& \lesssim\left(\left\|\partial_{v} \Delta_{t}^{-1} f_{0}\right\|_{L^{\infty} L^{2}}+\left\|f_{0}\right\|_{L^{\infty} H^{N}}\right)\left\|f_{\neq}\right\|_{L^{2} H^{N}}\left\|A f_{\neq}\right\|_{L^{2} L^{2}}
\end{aligned}
$$

Using (3.13) and Sobolev embedding, we have for $\delta$ sufficiently small (recall $u_{0}^{z}=-a \partial_{v} \Delta_{t}^{-1} f_{0}$ ),

$$
\begin{aligned}
\left|\mathcal{T}_{0}\right| & \lesssim\left(\left\|u_{0}^{z}\right\|_{L^{\infty} L^{2}}+\left\|f_{0}\right\|_{L^{\infty} H^{N}}\right)\left\|f_{\neq}\right\|_{L^{2} H^{N}}\left\|A f_{\neq}\right\|_{L^{2} L^{2}} \\
& \lesssim \varepsilon^{3} \nu^{-1 / 3} .
\end{aligned}
$$

Here in the last step we used the bootstrap hypotheses (3.16). This is now consistent with Proposition 3.8 provided $\varepsilon \ll \nu^{1 / 3}$ (which of course is weaker than the hypotheses $\varepsilon \ll \nu^{1 / 2}$ ). 
Next we estimate the source term $S$ in (3.17). Recall that $b=b(t, v)$ and thus

$$
S=\iint A\left(b \partial_{z} \Delta_{t}^{-1} f_{\neq}\right) A f_{\neq} d V d t .
$$

Via Plancherel's theorem, (2.6c), and (2.6f), there holds

$$
\begin{aligned}
& |S|=\left|\iint A\left(b \partial_{z} \Delta_{L}^{-1} \Delta_{L} \Delta_{t}^{-1} f_{\neq}\right) A f_{\neq} d V d t\right| \\
& =\frac{1}{4 \pi^{2}}\left|\sum_{k \neq 0} \iiint A(k, \xi) \hat{b}(\xi-\eta) \frac{i k}{k^{2}+|\eta-k t|^{2}}\left(\widehat{\Delta_{L} \Delta_{t}^{-1}} f_{\neq}\right)(k, \eta) A(k, \xi) \overline{\hat{f}}(k, \xi) d \eta d \xi d t\right| \\
& \lesssim \sum_{k \neq 0} \iiint\left|A(k, \xi) \hat{b}(\xi-\eta) \frac{-\dot{M}(k, \eta)}{M(k, \eta)}\left(\widehat{\Delta_{L} \Delta_{t}^{-1}} f_{\neq}\right)(k, \eta) A(k, \xi) \hat{f}(k, \xi)\right| d \eta d \xi d t \\
& \lesssim \sum_{k \neq 0} \iiint\left|A(k, \xi)\langle\xi-\eta\rangle \hat{b}(\xi-\eta) \sqrt{\frac{-\dot{M}(k, \eta)}{M(k, \eta)}}\left(\widehat{\Delta_{L} \Delta_{t}^{-1}} f_{\neq}\right)(k, \eta)\right| \\
& \times\left|\sqrt{\frac{-\dot{M}(k, \xi)}{M(k, \xi)}} A(k, \xi) \hat{f}(k, \xi)\right| d \eta d \xi d t \\
& \lesssim \sum_{k \neq 0} \iiint\left|\langle\xi-\eta\rangle^{N+1} \hat{b}(\xi-\eta) \sqrt{\frac{-\dot{M}(k, \eta)}{M(k, \eta)}} A(k, \eta)\left(\widehat{\Delta}_{L}{\widehat{\Delta_{t}^{-1}}}_{f \neq}\right)(k, \eta)\right| \\
& \times\left|\sqrt{\frac{-\dot{M}(k, \xi)}{M(k, \xi)}} A(k, \xi) \hat{f}(k, \xi)\right| d \eta d \xi d t .
\end{aligned}
$$

Young's convolution inequality followed by Lemma A.3 and (3.13) yields

$$
\begin{aligned}
|S| & \lesssim\|b\|_{H^{N+2}}\left\|\sqrt{-\dot{M} M}\langle D\rangle^{N} \Delta_{L} \Delta_{t}^{-1} f_{\neq}\right\|_{L^{2} L^{2}}\left\|\sqrt{-\dot{M} M}\langle D\rangle^{N} f_{\neq}\right\|_{L^{2} L^{2}} \\
& \lesssim \delta\left\|\sqrt{-\dot{M} M}\langle D\rangle^{N} f_{\neq}\right\|_{L^{2} L^{2}}^{2} .
\end{aligned}
$$

Hence, for $\delta$ sufficiently small, this term is absorbed on the left hand side of (3.17).

Finally, we estimate the diffusion error term $D E$ in (3.17). First divide into zero and non-zero modes,

$$
\begin{aligned}
D E & =\nu \iint A\left(\left(a^{2}-1\right) \partial_{v v} f_{0}\right) A f_{0} d V d t+\nu \iint A\left(\left(a^{2}-1\right) \partial_{v v}^{L} f_{\neq}\right) A f_{\neq} d V d t, \\
& =D E_{0}+D E_{\neq} .
\end{aligned}
$$

Integration by parts, followed by (3.10), (3.13), and a bound similar to (3.19), implies

$$
\begin{aligned}
\left|D E_{\neq}\right| & =\left|2 \nu \iint A\left(b \partial_{v}^{L} f_{\neq}\right) A f_{\neq} d V d t+\nu \iint A\left(\left(a^{2}-1\right) \partial_{v}^{L} f_{\neq}\right) \partial_{v}^{L} A f_{\neq} d V d t\right| \\
& \lesssim \delta \nu\left\|\nabla_{L} f_{\neq}\right\|_{L^{2} H^{N}}\left\|A f_{\neq}\right\|_{L^{2} L^{2}}+\delta \nu\left\|\nabla_{L} f_{\neq}\right\|_{L^{2} H^{N}}\left\|\nabla_{L} A f_{\neq}\right\|_{L^{2} L^{2}} \\
& \lesssim \delta \nu\left\|\nabla_{L} f_{\neq}\right\|_{L^{2} H^{N}}^{2}+\delta \nu\left\|A f_{\neq}\right\|_{L^{2} L^{2}}^{2} \\
& \lesssim \delta \nu \varepsilon^{2} \nu^{-1} \lesssim \delta \varepsilon^{2} .
\end{aligned}
$$


This is consistent with Proposition 3.8 provided $\delta$ is chosen sufficiently small (independently of $\nu$ and $\varepsilon$ ). For the zero mode term, we similarly integrate by parts and use (3.12) to obtain

$$
\begin{aligned}
\left|D E_{0}\right| & \lesssim \nu\|b\|_{L^{2} H^{N}}\left\|\partial_{v} f_{0}\right\|_{L^{2} H^{N}}\left\|f_{0}\right\|_{L^{\infty} H^{N}}+\nu\left\|a^{2}-1\right\|_{L^{\infty} H^{N}}\left\|\partial_{v} f_{0}\right\|_{L^{2} H^{N}}^{2} \\
& \lesssim \delta \varepsilon^{2},
\end{aligned}
$$

which is again consistent with Proposition 3.8 by choosing $\delta$ sufficiently small. This concludes improvement of (3.16a) for $\varepsilon$ and $\delta$ sufficiently small.

3.4.2. Velocity estimate. Here we improve (3.16b). By Lemmas 3.3 and 3.5 we have

$$
\frac{1}{2}\left\|u_{0}\right\|_{L^{2}} \leq\left\|\bar{v}_{0}\right\|_{L^{2}} \leq 2\left\|u_{0}\right\|_{L^{2}}
$$

provided $\delta$ is small enough. Thus, in order to prove (3.16b), we only need to prove the inequality in the original coordinates for $\bar{v}_{0}$.

Taking the $x$ average of (1.3a), we obtain the equation for $\bar{v}_{0}^{x}$

$$
\partial_{t} \bar{v}_{0}^{x}+\left(\bar{v} \cdot \nabla \bar{v}^{x}\right)_{0}-\nu \Delta \bar{v}_{0}^{x}=0
$$

where we used $\bar{v}_{0}^{y}=0$ due to the divergence-free condition. Multiplying the above equation by $\bar{v}_{0}^{x}$ and integrating over $y$ on $\mathbb{R}$, we obtain the energy estimate

$$
\frac{1}{2} \frac{d}{d t}\left\|\bar{v}_{0}^{x}\right\|_{L^{2}}^{2}+\nu\left\|\nabla \bar{v}_{0}^{x}\right\|_{L^{2}}^{2}=-\int\left(\bar{v} \cdot \nabla \bar{v}^{x}\right)_{0} \bar{v}_{0}^{x} d y .
$$

Integrating in time gives

$$
\begin{aligned}
\frac{1}{2}\left\|\bar{v}_{0}^{x}(t)\right\|_{L^{2}}^{2}+\nu\left\|\nabla \bar{v}_{0}^{x}(t)\right\|_{L^{2} L^{2}}^{2} & =\frac{1}{2}\left\|\bar{v}_{0}^{x}(0)\right\|_{L^{2}}^{2}-\iint\left(\bar{v} \cdot \nabla \bar{v}^{x}\right)_{0} \bar{v}_{0}^{x} d y d t \\
& =\frac{1}{2}\left\|\bar{v}_{0}^{x}(0)\right\|_{L^{2}}^{2}-\overline{\mathcal{T}} .
\end{aligned}
$$

In order to estimate the transport term $\overline{\mathcal{T}}$, we recall that $\bar{v}_{0}^{y}=0$, use the divergence free condition, and apply Plancherel's theorem to arrive at

$$
\begin{aligned}
\overline{\mathcal{T}} & =\iint \bar{v}_{0}^{x} \partial_{x} \bar{v}_{0}^{x} \bar{v}_{0} d y d t+\iint\left(\bar{v}_{\neq} \cdot \nabla \bar{v}_{\neq}^{x}\right)_{0} \bar{v}_{0}^{x} d y d t \\
& =\iint\left(\bar{v}_{\neq} \cdot \nabla \bar{v}_{\neq}^{x}\right)_{0} \bar{v}_{0}^{x} d y d t=\iint\left(\nabla \cdot\left(\bar{v}_{\neq} \bar{v}_{\neq}^{x}\right)\right)_{0} \bar{v}_{0}^{x} d y d t=\iint\left(\partial_{y}\left(\bar{v}_{\neq}^{y} \bar{v}_{\neq}^{x}\right)\right)_{0} \bar{v}_{0}^{x} d y d t .
\end{aligned}
$$

Physically this term corresponds to the transfer of kinetic energy from the non-zero modes to the zero mode. By changing coordinates back to $(z, v)$ inside the $x$ integral we obtain,

$$
\begin{aligned}
\left(\partial_{y}\left(\bar{v}_{\neq}^{y} \bar{v}_{\neq}^{x}\right)\right)_{0} & =-\int a\left(\partial_{v}-t \partial_{z}\right)\left(a\left(\partial_{v}-t \partial_{z}\right) \phi_{\neq} \partial_{z} \phi_{\neq}\right) d z \\
& =-\int a \partial_{v}\left(a\left(\partial_{v}-t \partial_{z}\right) \phi_{\neq} \partial_{z} \phi_{\neq}\right) d z .
\end{aligned}
$$

Therefore, by (3.13), Lemma 3.5, Lemma A.4, and the Sobolev embedding we have

$$
\begin{aligned}
|\overline{\mathcal{T}}| & \lesssim\left(\left\|\partial_{v} \partial_{v}^{L} \phi_{\neq}\right\|_{L^{2} L^{2}}\left\|\partial_{z} \phi_{\neq}\right\|_{L^{2} L^{\infty}}+\left\|\partial_{v}^{L} \phi_{\neq}\right\|_{L^{2} L^{\infty}}\left\|\partial_{v z} \phi_{\neq}\right\|_{L^{2} L^{2}}\right)\left\|\bar{v}_{0}^{x}\right\|_{L^{\infty} L^{2}} \\
& \lesssim\left\|f_{\neq}\right\|_{L^{2} H^{N}}^{2}\left\|\bar{v}_{0}^{x}\right\|_{L^{\infty} L^{2}} \\
& \lesssim \varepsilon^{3} \nu^{-1 / 3}
\end{aligned}
$$

which is sufficient to improve (3.16b) and hence conclude the proof of Proposition 3.8. Therefore, this also concludes the proof of Theorem 3.7. 


\section{Appendix A. Construction and properties of the multiplier $M$}

In this section we recall some of the technical tools regarding the Fourier multiplier $M$ from [BGM15c] and adapt them to our simpler setting.

LEMmA A.1. There exists a multiplier $M$ such that the conditions (2.6a)-(2.6e) hold for some constant $0<c<1$.

Proof of Lemma A.1. We consider a multiplier of the form $M=M_{1} M_{2}$ such that both $M_{1}$ and $M_{2}$ satisfy (2.6a). We choose $M_{1}$ such that for $k \neq 0$ it is determined by the ODE

$$
\begin{aligned}
-\frac{\dot{M}_{1}}{M_{1}} & =\frac{|k|}{k^{2}+|\xi-k t|^{2}} \\
M_{1}(0, k, \eta) & =1 .
\end{aligned}
$$

Similar multipliers appeared in [Zil14, BGM15a, BGM15b, BGM15c]. The above multiplier clearly satisfies (2.6c). Notice that for $k \neq 0$, there holds

$$
\frac{k^{2}+|\eta-k t|^{2}}{k^{2}+|\xi-k t|^{2}}=\frac{k^{2}+|\xi-k t+\eta-\xi|^{2}}{k^{2}+|\xi-k t|^{2}} \lesssim 1+|\eta-\xi|^{2},
$$

and hence (2.6f) holds for $M_{1}$. A direct computation shows that

$$
M_{1}(t, k, \xi)=\exp \left(-\int_{0}^{t} \frac{|k|}{k^{2}+|\xi-k s|^{2}} d s\right),
$$

which implies (2.6b) holds for $M_{1}$. Taking the derivative of $M_{1}$ with respect to $\xi$ gives

$$
\left|\frac{\partial_{\xi} M_{1}(k, \xi)}{M_{1}(k, \xi)}\right|=\left|\int_{0}^{t} \frac{2|k|(\xi-k s)}{\left(k^{2}+|\xi-k s|^{2}\right)^{2}} d s\right| \leq \frac{2}{|k|^{2}} \int_{0}^{t} \frac{1}{\left(1+|\xi / k-s|^{2}\right)} d s,
$$

which proves that (2.6d) holds for $M_{1}$. Note here that $k \neq 0$ implies $|k| \geq 1$.

Next, we define $M_{2}$ by the differential equation (for $k \neq 0$ ),

$$
\begin{aligned}
-\frac{\dot{M}_{2}}{M_{2}} & =\frac{\nu^{1 / 3}}{\left(\nu^{1 / 3}|t-\xi / k|\right)^{2}+1} \\
M_{2}(0, k, \eta) & =1 .
\end{aligned}
$$

This multiplier was introduced in [BGM15c]. Similarly to $M_{1}$, we deduce that (2.6b), (2.6d), and (2.6f) all hold for $M_{2}$ (and hence also for $M=M_{1} M_{2}$ ). Since for $k \neq 0$ we have that

$$
1 \lesssim \nu^{1 / 3}|k, \xi-k t| \quad \text { if } \nu^{-1 / 3} \leq\left|t-\frac{\xi}{k}\right|
$$

and

$$
1 \lesssim \frac{1}{\left(\nu^{1 / 3}|t-\xi / k|\right)^{2}+1} \quad \text { if } \nu^{-1 / 3} \geq\left|t-\frac{\xi}{k}\right|,
$$

inequality (2.6e) holds for $M_{2}$. Therefore, the multiplier $M$ we constructed satisfies conditions (2.6a)-(2.6e), completing the proof.

REMARK A.2. In condition (2.6e), the power $\nu^{-1 / 6}$ in front of the multiplier is sharp in the sense that $1 / 6$ is the smallest sacrifice we need to make to bound the expression on the right side from below by a constant. In fact, if we make $M_{2}$ to be positive constants independent of $\nu$ when 
$|t-\xi / k| \geq \nu^{-1 / 3}$, then the size of $\dot{M}_{2}$ should be approximately $\nu^{1 / 3}$. Hence, if we did not need (2.6f) or (2.6d), one could construct $M_{2}$ to satisfy (2.6e) with

$$
\begin{aligned}
& \dot{M}_{2}=0 \quad \text { if } \nu^{-1 / 3} \geq\left|t-\frac{\xi}{k}\right| \\
& \dot{M}_{2}=-\frac{1}{2} \nu^{1 / 3} \quad \text { if } \nu^{-1 / 3} \geq\left|t-\frac{\xi}{k}\right| .
\end{aligned}
$$

We need the following lemma to commute $\sqrt{-\dot{M} M}$ with $\Delta_{L} \Delta_{t}^{-1}$, the latter of which is not a Fourier multiplier.

Lemma A.3. Let $f \in H^{N}$ and $N>1$. Then the following estimate holds for $\delta$ sufficiently small,

$$
\left\|\sqrt{-\dot{M} M} \Delta_{L} \Delta_{t}^{-1} f_{\neq}\right\|_{H^{N}} \lesssim\left\|\sqrt{-\dot{M} M} f_{\neq}\right\|_{H^{N}}
$$

Proof of Lemma A.3. Using the equality

$$
\Delta_{L}=\Delta_{t}-\left(a^{2}-1\right) \partial_{v v}^{L}-b \partial_{v}^{L}
$$

we have

$$
\begin{aligned}
\left\|\sqrt{-\dot{M} M} \Delta_{L} \Delta_{t}^{-1} f_{\neq}\right\|_{H^{N}} \lesssim & \left\|\sqrt{-\dot{M} M} f_{\neq}\right\|_{H^{N}}+\left\|\sqrt{-\dot{M} M}\left(\left(a^{2}-1\right) \partial_{v v}^{L} \Delta_{t}^{-1} f_{\neq}\right)\right\|_{H^{N}} \\
& +\left\|\sqrt{-\dot{M} M}\left(b \partial_{v}^{L} \Delta_{t}^{-1} f_{\neq}\right)\right\|_{H^{N}} .
\end{aligned}
$$

By (2.6f), $N>1$, and (3.13), we deduce

$$
\begin{aligned}
\left\|\sqrt{-\dot{M} M}\left(\left(a^{2}-1\right) \partial_{v v}^{L} \Delta_{t}^{-1} f_{\neq}\right)\right\|_{H^{N}} & =\left\|\sqrt{-\dot{M} M}\left(\left((a-1)^{2}-2(a-1)\right) \partial_{v v}^{L} \Delta_{t}^{-1} f_{\neq}\right)\right\|_{H^{N}} \\
& \lesssim\|a-1\|_{H^{N+1}}\left\|\sqrt{-\dot{M} M} \Delta_{L} \Delta_{t}^{-1} f_{\neq}\right\|_{H^{N}} \\
& \lesssim \delta\left\|\sqrt{-\dot{M} M} \Delta_{L} \Delta_{t}^{-1} f_{\neq}\right\|_{H^{N}},
\end{aligned}
$$

and similarly

$$
\begin{aligned}
\left\|\sqrt{-\dot{M} M}\left(b \partial_{v}^{L} \Delta_{t}^{-1} f_{\neq}\right)\right\|_{H^{N}} & \lesssim\|b\|_{H^{N+1}}\left\|\sqrt{-\dot{M} M} \Delta_{L} \Delta_{t}^{-1} f_{\neq}\right\|_{H^{N}} \\
& \lesssim \delta\left\|\sqrt{-\dot{M} M} \Delta_{L} \Delta_{t}^{-1} f_{\neq}\right\|_{H^{N}}
\end{aligned}
$$

Since $\delta \ll 1$, the result follows from (A.1) immediately.

The following lemma is proved in the same manner as Lemma A.3, although slightly simpler. In particular, this lemma shows that $\Delta_{L} \Delta_{t}^{-1}$ can be approximately treated as the identity for $\delta$ sufficiently small.

Lemma A.4. Let $f \in H^{N}$ and $N>1$. Then the following estimate holds for $\delta$ sufficiently small,

$$
\left\|\Delta_{L} \Delta_{t}^{-1} f_{\neq}\right\|_{H^{N}} \lesssim\left\|f_{\neq}\right\|_{H^{N}}
$$

The following estimate applies to the zero mode of the velocity field.

Lemma A.5. Let $f$ be such that $f_{0} \in H^{N}, N>1$, and $\partial_{v} \Delta_{t}^{-1} f_{0} \in L^{2}$. Then for $\delta$ sufficiently small, there holds

$$
\left\|\partial_{v v} \Delta_{t}^{-1} f_{0}\right\|_{H^{N}} \lesssim\left\|f_{0}\right\|_{H^{N}}+\delta\left\|\partial_{v} \Delta_{t}^{-1} f_{0}\right\|_{L^{2}}
$$


Proof of Lemma A.5. Since

$$
\partial_{v v} \Delta_{t}^{-1} f_{0}=\left(\Delta_{t}-\left(a^{2}-1\right) \partial_{v v}-b \partial_{v}\right) \Delta_{t}^{-1} f_{0},
$$

we have by $N>1$ and (3.13) (interpolating $H^{N}$ between $L^{2}$ and $H^{N+1}$ ),

$$
\begin{aligned}
\left\|\partial_{v v} \Delta_{t}^{-1} f_{0}\right\|_{H^{N}} & \lesssim\left\|f_{0}\right\|_{H^{N}}+\delta\left\|\partial_{v v} \Delta_{t}^{-1} f_{0}\right\|_{H^{N}}+\delta\left\|\partial_{v} \Delta_{t}^{-1} f_{0}\right\|_{H^{N}} \\
& \lesssim\left\|f_{0}\right\|_{H^{N}}+\delta\left\|\partial_{v v} \Delta_{t}^{-1} f_{0}\right\|_{H^{N}}+\delta\left\|\partial_{v} \Delta_{t}^{-1} f_{0}\right\|_{L^{2}} .
\end{aligned}
$$

For $\delta \ll 1$, we may absorb the second term on the right side and we obtain the desired result.

\section{Acknowledgments}

The work of JB was partially supported by NSF grant DMS-1462029, and by an Alfred P. Sloan Research Fellowship. The work of VV was partially supported by NSF grant DMS-1514771 and by an Alfred P. Sloan Research Fellowship. The work of FW was partially supported by NSF grant DMS-1514771.

\section{References}

[Ali01] S. Alinhac. The null condition for quasilinear wave equations in two space dimensions I. Invent. Math., 145(3):597-618, 2001.

[BCZ15] J. Bedrossian and M. Coti Zelati. Enhanced dissipation, hypoellipticity, and anomalous small noise inviscid limits in shear flows. arXiv:1510.08098, 2015.

[BCZGH15] J. Bedrossian, M. Coti Zelati, and N. Glatt-Holtz. Invariant measures for passive scalars in the small noise inviscid limit. arXiv:1505.07356, 2015.

[BGM15a] J. Bedrossian, P. Germain, and N. Masmoudi. Dynamics near the subcritical transition of the 3D Couette flow I: Below threshold. arXiv:1506.03720, 2015.

[BGM15b] J. Bedrossian, P. Germain, and N. Masmoudi. Dynamics near the subcritical transition of the 3D Couette flow II: Above threshold. arXiv:1506.03721, 2015.

[BGM15c] J. Bedrossian, P. Germain, and N. Masmoudi. On the stability threshold for the 3D Couette flow in Sobolev regularity. arXiv:1511.01373, 2015.

[BL94] A.J. Bernoff and J.F. Lingevitch. Rapid relaxation of an axisymmetric vortex. Phys. Fluids, 6(3717), 1994.

[BM13] J. Bedrossian and N. Masmoudi. Inviscid damping and the asymptotic stability of planar shear flows in the 2D Euler equations. Publications mathématiques de l'IHÉS, pages 1-106, 2013.

[BMM13] J. Bedrossian, N. Masmoudi, and C. Mouhot. Landau damping: paraproducts and Gevrey regularity. To appear in Annals of PDE, 2013.

[BMV16] J. Bedrossian, N. Masmoudi, and V. Vicol. Enhanced dissipation and inviscid damping in the inviscid limit of the Navier-Stokes equations near the 2D Couette flow. Arch. Rat. Mech. Anal., 216(3):1087-1159, 2016.

[BW13] M. Beck and C.E. Wayne. Metastability and rapid convergence to quasi-stationary bar states for the twodimensional Navier-Stokes equations. Proc. Royal Soc. of Edinburgh: Sec. A Mathematics, 143(05):905927, 2013.

[CKRZ08] P. Constantin, A. Kiselev, L. Ryzhik, and A. Zlatoš. Diffusion and mixing in fluid flow. Ann. of Math. (2), 168:643-674, 2008.

[CM98] E. Caglioti and C. Maffei. Time asymptotics for solutions of Vlasov-Poisson equation in a circle. J. Stat. Phys., 92(1/2), 1998.

[DN94] B. Dubrulle and S. Nazarenko. On scaling laws for the transition to turbulence in uniform-shear flows. Euro. Phys. Lett., 27(2):129, 1994.

[DR81] P.G. Drazin and W.H. Reid. Hydrodynamic stability. Cambridge U. Press, Cambridge, 1981.

[GGN09] I. Gallagher, T. Gallay, and F. Nier. Spectral asymptotics for large skew-symmetric perturbations of the harmonic oscillator. International Mathematics Research Notices, page rnp013, 2009.

[IKT13] H. Inci, T. Kappeler, and P. Topalov. On the regularity of the composition of diffeomorphisms. Mem. Amer. Math. Soc., 226(1062):vi+60, 2013.

[Kel87] Lord Kelvin. Stability of fluid motion-rectilinear motion of viscous fluid between two parallel plates. Phil. Mag., (24):188, 1887. 
[LB01] M. Latini and A.J. Bernoff. Transient anomalous diffusion in Poiseuille flow. J. of Fluid Mech., 441:399411, 2001.

[Lun82] T.S. Lundgren. Strained spiral vortex model for turbulent fine structure. Phys. of Fl., 25:2193, 1982.

[LZ11] Z. Lin and C. Zeng. Inviscid dynamic structures near Couette flow. Arch. Rat. Mech. Anal., 200:1075-1097, 2011.

[MV11] C. Mouhot and C. Villani. On Landau damping. Acta Math., 207(1):29-201, 2011.

[Orr07] W. Orr. The stability or instability of steady motions of a perfect liquid and of a viscous liquid, Part I: a perfect liquid. Proc. Royal Irish Acad. Sec. A: Math. Phys. Sci., 27:9-68, 1907.

[RSBH98] S.C. Reddy, P.J. Schmid, J.S. Baggett, and D.S. Henningson. On stability of streamwise streaks and transition thresholds in plane channel flows. J. of Fluid Mech., 365:269-303, 1998.

[RY83] P.B. Rhines and W.R. Young. How rapidly is a passive scalar mixed within closed streamlines? J. of Fluid Mech., 133:133-145, 1983.

[Ryu99] D.D. Ryutov. Landau damping: half a century with the great discovery. Plasma physics and controlled fusion, 41(3A):A1, 1999.

[SH01] P.J. Schmid and D.S. Henningson. Stability and transition in shear flows, volume 142. Springer, 2001.

[VDPS15] J. Vukadinovic, E. Dedits, A. C. Poje, and T. Schäfer. Averaging and spectral properties for the 2D advection-diffusion equation in the semi-classical limit for vanishing diffusivity. Phys. D, 310:1-18, 2015.

[WZZ15] D. Wei, Z. Zhang, and W. Zhao. Linear inviscid damping for a class of monotone shear flow in Sobolev spaces. Preprint, arxiv:1509.08228, 2015.

[Yag12] A.M. Yaglom. Hydrodynamic Instability and Transition to Turbulence, volume 100. Springer, 2012.

[YD02] J.H. Yu and C.F. Driscoll. Diocotron wave echoes in a pure electron plasma. IEEE Trans. Plasma Sci., 30(1), 2002.

[YDO05] J.H. Yu, C.F. Driscoll, and T.M. O`Neil. Phase mixing and echoes in a pure electron plasma. Phys. of Plasmas, 12(055701), 2005.

[Zi114] C. Zillinger. Linear inviscid damping for monotone shear flows. arXiv preprint arXiv:1410.7341, 2014.

[Zla10] A. Zlatoš. Diffusion in fluid flow: dissipation enhancement by flows in 2D. Comm. Partial Differential Equations, 35(3):496-534, 2010.

Department of Mathematics, University of Maryland, College Park, MD 20742

E-mail address: jacob@cscamm.umd.edu

Department of Mathematics, Princeton University, Princeton, NJ 08544

E-mail address: vvicol@math.princeton.edu

Department of Mathematics, University of Southern California, Los Angeles, CA 90089

E-mail address: wang 828 Cusc. edu 